\title{
Article
}

\section{Music Therapy Is Effective during Sleep in Preterm Infants}

\author{
Susann Kobus *(D), Marlis Diezel, Monia Vanessa Dewan, Britta Huening, Anne-Kathrin Dathe (D), \\ Ursula Felderhoff-Mueser and Nora Bruns (D)
}

\author{
Clinic for Pediatrics I, Essen University Hospital, University of Duisburg-Essen, 45147 Essen, Germany; \\ marlis.diezel@gmail.com (M.D.); Monia.Dewan@uk-essen.de (M.V.D.); Britta.Huening@uk-essen.de (B.H.); \\ Anne-Kathrin.Dathe@uk-essen.de (A.-K.D.); Ursula.Felderhoff@uk-essen.de (U.F.-M.); \\ nora.bruns@uk-essen.de (N.B.) \\ * Correspondence: susann.kobus@uk-essen.de
}

check for updates

Citation: Kobus, S.; Diezel, M.; Dewan, M.V.; Huening, B.; Dathe, A.-K.; Felderhoff-Mueser, U.;

Bruns, N. Music Therapy Is Effective during Sleep in Preterm Infants. Int. J. Environ. Res. Public Health 2021, 18 , 8245. https://doi.org/10.3390/ ijerph18168245

Academic Editors: Łucja Bieleninik and Mark Ettenberger

Received: 27 June 2021

Accepted: 30 July 2021

Published: 4 August 2021

Publisher's Note: MDPI stays neutral with regard to jurisdictional claims in published maps and institutional affiliations.

Copyright: (c) 2021 by the authors. Licensee MDPI, Basel, Switzerland. This article is an open access article distributed under the terms and conditions of the Creative Commons Attribution (CC BY) license (https:/ / creativecommons.org/licenses/by/ $4.0 /)$.

\begin{abstract}
Recent research found evidence supporting music therapy for preterm infants to stabilize vital signs and possibly promote neurodevelopment. Even though preterm infants spend a considerable amount of time sleeping, the effectiveness of music therapy during sleep has not been studied. The aim of this study was to investigate the effect of music therapy on preterm infants' vital signs with respect to the state of wakefulness. The first 20 consecutive infants born with $<32$ weeks' gestational age (GA) from the intervention group of an ongoing randomized controlled trial received live music therapy twice a week until hospital discharge. The heart rate, respiratory rate, oxygen saturation, and state of wakefulness were recorded before and after therapy. We observed significantly lower heart and respiratory rates and higher oxygen saturation after live music therapy sessions in general (mean differences -4.9 beats per $\mathrm{min} ;-7.0$ breaths per min and $+1.5 \%$, respectively). When music therapy was applied during sleep, respiratory rates significantly lowered by 8.8 breaths per min and oxygen saturation increased by $1.6 \%$, whereas in the awake state the vital parameters did not significantly change (heart rate -5.2 beats per min; respiratory rate +0.6 breaths per min and oxygen saturation $+1.0 \%$ ). Music therapy stabilized the respiratory rates and oxygen saturations in sleeping preterm infants.
\end{abstract}

Keywords: music therapy; preterm infants; sleep; neonatal intensive care unit; premature infants

\section{Introduction}

From a gestational age of 26 weeks, the human fetus can perceive auditory stimuli [1]. Maturation of the auditory system is influenced by the acoustic environment of an infant and is the basis for later language development, learning and memory formation [2]. In infants born prematurely, this maturational process is disrupted. Preterm birth coincides with a period of rapid brain development, making the infant's brain highly vulnerable but responsive at the same time to neuroprotective interventions [1].

A promising family-integrated early intervention to improve infant development, parental well-being, and bonding is music therapy [3]. It is feasible and well-tolerated by infants, parents, and staff even after extremely preterm birth $[4,5]$. Parents and staff perceived music therapy as valuable for development-promoting care in the neonatal intensive care unit [5]. Infants were not overstimulated by live-performed music therapy [5]. Furthermore, music therapy attenuates infants' stress in the neonatal intensive care unit, even though the strength and sustainability of the effect remains unclear [6]. Several studies showed that music therapy had a stabilizing and relaxing effect on the preterm infant's general behavioral status, sleep patterns, and vital signs [7-11]. Live music and live sung lullabies were superior compared to recorded music in reducing the heart rate and improving behavioral scores [12].

Even though newborns and preterm infants spend approximately two thirds of the daytime sleeping during their first weeks of life [13], we found no study that assessed the 
effect of music therapy during sleep compared to wakefulness. From our own experience, some staff members and parents raise concerns that music therapy is not effective when applied during sleep. The aim of this study was to assess if live music therapy affected preterm infants' vital signs when applied during sleep.

\section{Materials and Methods}

\subsection{Study Design}

The study was designed as a prospective, randomized controlled clinical trial which is still ongoing. Infants were randomly assigned to either music therapy (intervention group) or no music therapy (control group). There were no differences between the groups in medical care. To investigate the effects of music therapy on vital signs we analyzed the protocols of 307 music therapy sessions of the first 20 consecutive infants that had been allocated to the intervention group.

\subsection{Eligibility and Recruitment}

Infants born before 32 weeks' gestation at the University Hospital Essen between October 2018 and November 2019 were eligible for the study. Because the study was designed to examine the neurodevelopment outcome later in the course, exclusion criteria were congenital hearing disorders, periventricular haemorrhagic infarction, cerebral malformations, and underlying diseases that impair neurological development.

Parental informed consent was obtained during the first week of life, at a minimum age of $72 \mathrm{~h}$. The study was approved by the local ethics committee of the Medical Faculty of the University of Duisburg-Essen (18-8035-BO).

\subsection{Intervention}

Music therapy was performed twice weekly in clinically stable patients from the second week of life until discharge. The timing of each therapy session was coordinated by the music therapist, nursing staff and parents. During music therapy, the infant remained in the same position as before therapy, e.g., the incubator, heated cod or parent's arm or breast during kangaroo care.

Each session consisted of individual, improvised singing by the music therapist and/or the use of the instrument sansula. Improvised singing was guided by the infant's breathing and reactions, beginning with humming tones that were followed by tone sequences with improvised text. The sansula consists of a wooden ring covered with an eardrum, on which a small kalimba is attached. It creates a space-filling, long-lasting and soft sound.

Music therapy was carried out for each child individually at a low volume and could not be perceived by other patients treated in the intensive care unit. Vital signs (heart rate, respiratory rate, and oxygen saturation), wakefulness, and physical contact during the session were documented in a protocol before and after each therapy session. Clinical data were retrieved from the patients' medical record.

\subsection{Statistical Analysis}

Quantitative variables are presented as the mean and 95\% confidence intervals or standard deviation, respectively; for qualitative factors, absolute and relative frequencies are given. Therapy sessions were stratified depending on the corrected gestational age at intervention into the age groups 24 to 27 weeks, 28 to 31 weeks, 32 to 35 weeks, 36 to 39 weeks and 40 to 43 weeks to assess the plausibility of our data. Depending on the infants wakefulness before and after therapy, we classified infants into four categories (stayed asleep, fell asleep, woke up and stayed awake).

The effect sizes for pre- and post-therapy vital sign values were calculated as suggested by Maier-Riehle for single group pre-post study designs [14]. All statistical calculations were performed using SAS Enterprise guide 7.1. (SAS Institute Inc., Cary, NC, USA). $p$ values $<0.05$ were considered statistically significant. 


\section{Results}

\subsection{Patients}

Seventy-eight infants at $<32$ weeks' gestational age were treated at the University Hospital Essen during the study period. Forty infants were included into the main study, 20 in the therapy group and 20 in the control group. The clinical characteristics of the included patients in the therapy and the control group are presented in Table 1.

Table 1. Clinical characteristics of the included patients in the therapy and the control group.

\begin{tabular}{ccc}
\hline & Therapy Group $(\boldsymbol{n = 2 0 )}$ & Control Group $(\boldsymbol{n}=\mathbf{2 0})$ \\
\hline Male, $n$ & $12(60 \%)$ & $8(40 \%)$ \\
Gestattional age (weeks) & $28.6( \pm 2.4)$ & $28.9(2.7)$ \\
gestation age (weeks), range & $23+6-31+4$ & $23+2-31+6$ \\
Birth weight (g) & $1059( \pm 389)$ & $1201( \pm 404)$ \\
Birth weight (g), range & $340-1700$ & $580-2120$ \\
Died, $n$ (age at death) & $1(9$ days) & $1(152$ days) \\
APGAR score at 10 min & $8.7( \pm 0.7)$ & $8.7( \pm 1.0)$ \\
APGAR score at min, range & $7.0-10.0$ & $6.0-10.0$ \\
Early onset sepsis, $n$ & $5(25 \%)$ & $4(20 \%)$ \\
Late onset sepsis, $n$ & $4(20 \%)$ & $5(25 \%)$ \\
Bronchopulmonary dypslasia (mild), $n$ & $1(5 \%)$ & $2(10 \%)$ \\
Bronchopulmonary dypslasia (severe), $n$ & $1(5 \%)$ & $1(5 \%)$ \\
Intraventricular hemorrhage ${ }^{\circ} \mathrm{I}-\mathrm{II}, n$ & $4(20 \%)$ & $3(15 \%)$ \\
Intraventricular hemorrhage ${ }^{\circ} \mathrm{III}, n$ & $1(5 \%)$ & 0 \\
Necrotizing enterocolitis, $n$ & $1(5 \%)$ & 0 \\
Patent ductus arteriosus (total), $n$ & $11(55 \%)$ & $7(35 \%)$ \\
Medical therapy, $n$ & $10(50 \%)$ & $7(35 \%)$ \\
Surgery, $n$ & $1(5 \%)$ & 0 \\
Antibiotic treatment, days & $8.7(10.8)$ & $8.4(17.7)$
\end{tabular}

Notes. SD = standard deviation. APGAR = Appearance, Pulse, grimace, Activity, and Respiration. Data are presented as the mean (standard deviation) if not indicated otherwise.

Thirty-eight infants were not included. The reasons for exclusion were birth in another hospital $(n=3 ; 8 \%)$, death before recruitment $(n=8 ; 21 \%)$, transfer to another hospital $(n=2 ; 6 \%)$, periventricular hemorrhagic infarction $(n=1 ; 3 \%)$, maternal critical illness $(n=2 ; 6 \%)$, insufficient german language skills to understand objectives of the study $(n=3$; $8 \%)$, and lack of interest to participate $(n=18 ; 47 \%)$. Excluded infants were born at a mean gestational age of $28+0$ weeks (range $22+4$ to $31+3$ weeks) with a mean weight of $1056 \pm 467 \mathrm{~g}$ (range 210 to $1860 \mathrm{~g}$ ).

\subsection{Music Therapy Sessions}

307 music therapy sessions were conducted in the therapy group between gestational ages of $24+0$ and $43+0$ weeks (Table 2). Forty-seven sessions were conducted with the infant awake and 150 during sleep. The mean duration of each music therapy session was $19.3 \pm 4.3 \mathrm{~min}$ (range 10 to $50 \mathrm{~min}$ ). 
Table 2. Heart rate, respiratory rate and oxygen saturation during music therapy sessions.

\begin{tabular}{|c|c|c|c|c|c|c|}
\hline Vital Sign & $\begin{array}{c}\text { Corrected Gestational } \\
\text { Age, Weeks }\end{array}$ & Sessions, $n$ & Mean before Therapy $(95 \% \mathrm{CI})$ & Mean after Therapy $(95 \%$ CI) & Mean Difference $(95 \% \mathrm{CI})$ & Effect Size \\
\hline \multirow[t]{6}{*}{ Heart rate (beats per min) } & all & 307 & $163.7(162.1-165.4)$ & $158.8(157.1-160.5)$ & $-4.9(-6.6-(-3.3))$ & -0.33 \\
\hline & $24-27$ & 14 & $169.8(163.2-176.4)$ & $170.7(165.1-176.3)$ & $0.9(-4.8-6.6)$ & 0.08 \\
\hline & $28-31$ & 70 & $169.7(166.8-172.6)$ & $169.0(166.1-171.9)$ & $-0.7(-4.0-2.6)$ & -0.06 \\
\hline & $32-35$ & 139 & $164.4(162.0-166.7)$ & $157.7(155.4-160.0)$ & $-6.7(-9.0-(-4.4))$ & -0.48 \\
\hline & $36-39$ & 69 & $157.5(153.5-161.5)$ & $151.8(148.5-155.2)$ & $-5.6(-9.5-(-1.8))$ & -0.34 \\
\hline & $40-43$ & 15 & $153.5(143.6-163.3)$ & $142.9(133.8-151.9)$ & $-10.6(-23.3-2.1)$ & 0.60 \\
\hline Respiratory rate, & all & 300 & $53.3(51.4-55.2)$ & $46.3(44.6-47.9)$ & $-7.0(-9.2-(-4.8))$ & -0.42 \\
\hline \multirow{4}{*}{ (breaths per min) } & $28-31$ & 70 & $55.8(51.7-59.9)$ & $47.1(43.3-50.9)$ & $-8.7(-14.0-(-3.3))$ & -0.51 \\
\hline & $32-35$ & 139 & $53.1(50.4-55.9)$ & $45.9(43.5-48.3)$ & $-7.3(-10.3-(-4.2))$ & -0.44 \\
\hline & $36-39$ & 68 & $50.4(46.4-54.3)$ & $45.1(41.6-48.6)$ & $-5.3(-10.0-(-0.6))$ & -0.32 \\
\hline & $40-43$ & 15 & $52.5(42.3-62.6)$ & $49.3(41.3-57.2)$ & $-3.2(-14.6-8.2)$ & -0.17 \\
\hline \multirow[t]{5}{*}{$\mathrm{SaO}_{2}(\%)$} & all & 306 & $95.1(94.6-95.7)$ & $96.6(96.2-97.1)$ & $1.5(1.0-2.0)$ & 0.31 \\
\hline & $24-27$ & 14 & $89.4(86.5-92.4)$ & $88.9(86.8-91.0)$ & $-0.5(-3.8-2.8)$ & -0.10 \\
\hline & $28-31$ & 70 & $93.5(92.2-94.7)$ & 94.7 (93.7-95.7) & $1.2(-0.2-2.6)$ & 0.23 \\
\hline & $32-35$ & 139 & $96.0(95.4-96.7)$ & 97.6 (97.2-98.1) & $1.6(1.0-2.2)$ & 0.41 \\
\hline & $36-39$ & 68 & $96.1(95.0-97.3)$ & $98.0(97.4-98.7)$ & $1.9(1.0-2.8)$ & 0.40 \\
\hline
\end{tabular}




\subsection{Analyses by Gestational Age}

\subsubsection{Heart Rate}

We found an overall decrease in the heart rate after therapy of 4.9 beats per $\min (95 \%$ CI 6.6-3.3) (Table 2). The baseline heart rate before therapy decreased with an increasing corrected gA (Figure 1a). Significant decreases could be observed in infants $>32$ weeks' corrected gA (Table 2 and Figure 1a). Accordingly, the calculated effect sizes increased with the gA.

A

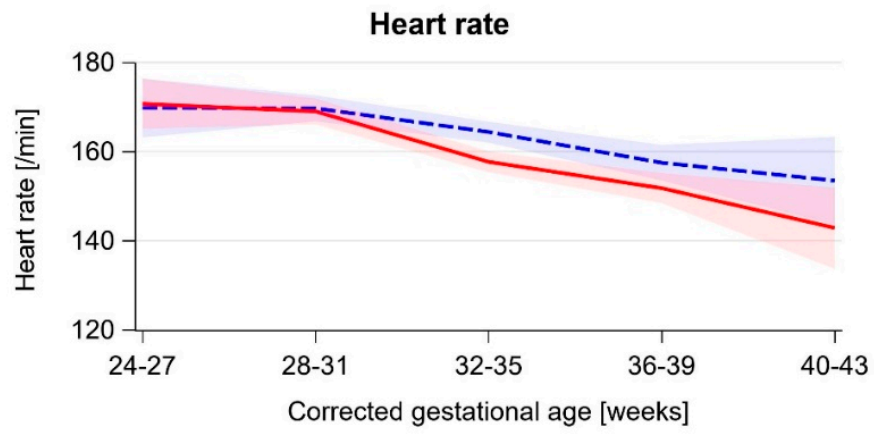

B

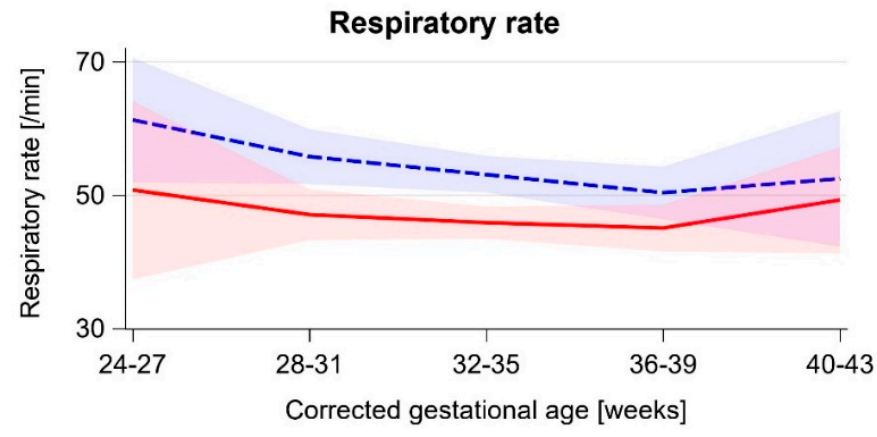

C

Oxygen saturation

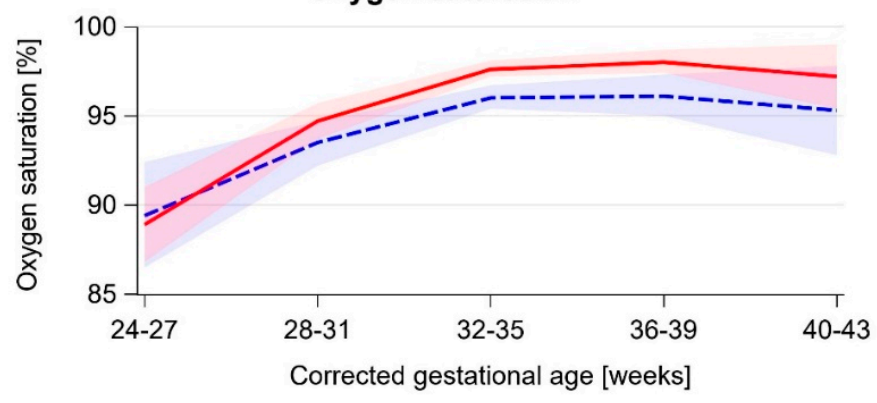

Figure 1. Baseline and post-therapy vital signs by corrected gestational age. Blue dashed line: mean baseline value, red solid line: mean post-therapy value, blue and red bands: corresponding 95\% confidence intervals. (A) Heart rate. (B) Respiratory rate. (C) Oxygen saturation.

\subsubsection{Respiratory Rate}

The overall decrease in respiratory rates was 7.0 breaths per min (95\% CI 9.2-4.8) (Table 2). The baseline respiratory rate before therapy decreased with an increasing gA but rose again at $>40$ weeks corrected gA (Figure $1 b$ ). Of the 15 therapy sessions at $>40$ weeks corrected gA, five (33\%) were performed in an infant with bronchopulmonary dysplasia.

Subanalyses showed larger absolute differences and stronger calculated effect sizes at lower corrected gAs (Table 2 and Figure 1b). 


\subsubsection{Oxygen Saturation}

We found an increase of 1.5\% (95\% CI 1.0-2.0) for all infants when comparing values before and after therapy (Table 2). The baseline oxygen saturation increased with a corrected gA until term equivalent age (Figure 1c). Stratification showed that infants at $>28$ weeks' corrected gA had higher $\mathrm{SaO}_{2}$ after therapy (Table 2 and Figure 1c). In infants at $>40$ weeks, the baseline and post-therapy $\mathrm{SaO}_{2}$ were lower. The calculated effect sizes increased with the $\mathrm{gA}$.

\subsection{Analyses by State of Wakefulness}

Out of 307 sessions, $150(49 \%)$ were performed while infants were asleep. During $79(26 \%)$ sessions infants fell asleep during music therapy, during $31(10 \%)$ they woke up, and during $47(15 \%)$ they stayed awake. The corrected gestational ages did not differ significantly between states of wakefulness (Table 3).

\subsubsection{Heart Rate}

Infants who fell asleep showed a decrease in heart rates of 14.2 beats per min (95\% CI 17.5-10.9) with non-overlapping confidence intervals for baseline and post-therapy values. The changes in the heart rates in the other groups were less pronounced (Table 3, Figure 2a). The calculated effect sizes were strongest in infants who fell asleep $(-1.00)$ and who stayed awake $(-0.30)$.

A

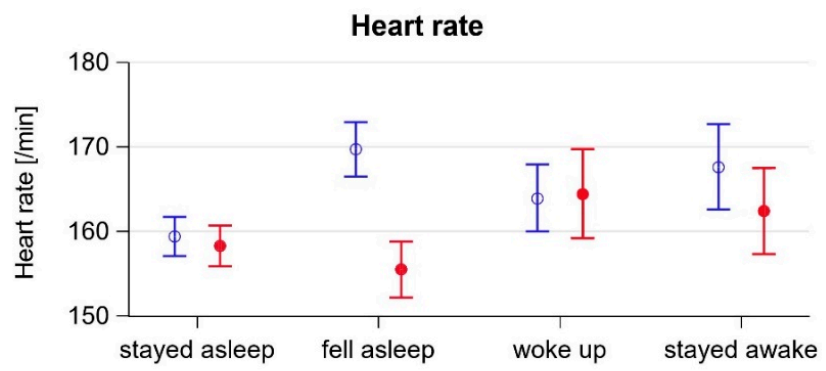

B

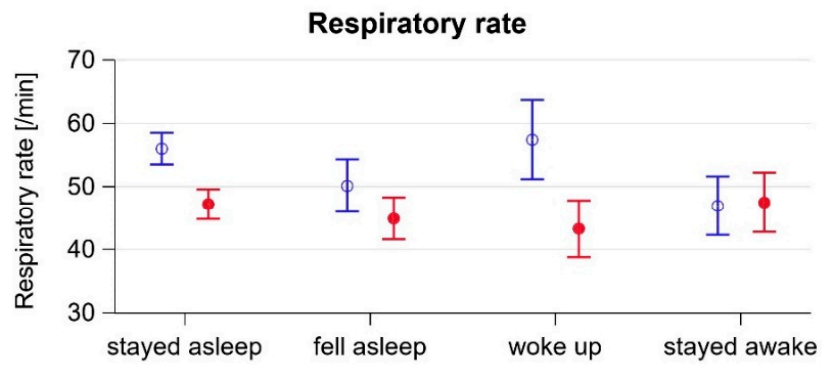

C

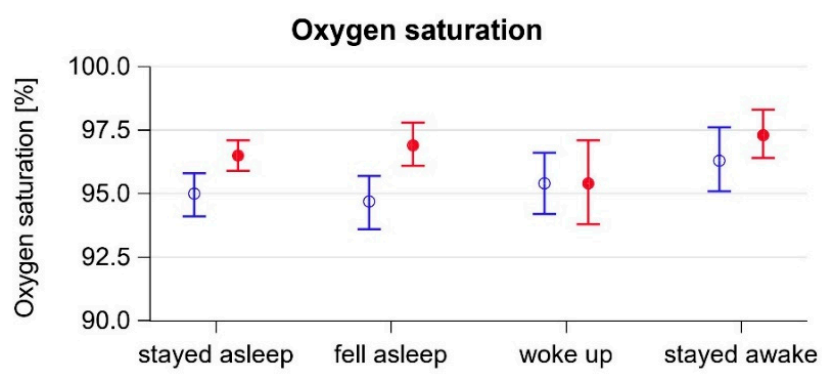

Figure 2. Baseline and post-therapy vital signs by states of wakefulness. Blue empty circle: mean baseline value, red filled circle: mean post-therapy value, blue and red error bars: corresponding $95 \%$ confidence intervals. (A) Heart rate. (B) Respiratory rate. (C) Oxygen saturation. 
Table 3. Response to music therapy by evolution of wakefulness during music therapy sessions.

\begin{tabular}{|c|c|c|c|c|c|c|c|}
\hline Vital Sign & Wakefulness & $\begin{array}{l}\text { Sessions } \\
\quad(n)\end{array}$ & $\begin{array}{c}\text { Mean Corrected Gestational } \\
\text { Age }(95 \% \mathrm{CI})\end{array}$ & $\begin{array}{c}\text { Mean before } \\
\text { Therapy }(95 \% \text { CI) }\end{array}$ & $\begin{array}{c}\text { Mean after Therapy } \\
(95 \% \mathrm{CI})\end{array}$ & $\begin{array}{l}\text { Mean Difference } \\
(95 \% \mathrm{CI})\end{array}$ & Effect Size \\
\hline \multirow[t]{3}{*}{ Heart rate (beats per min) } & stayed asleep & 150 & $33.6(33.1-34.2)$ & $159.4(157.1-161.7)$ & $158.3(155.9-160.7)$ & $-1.1(-3.0-0.8)$ & -0.08 \\
\hline & fell asleep & 79 & $34.3(33.5-35.1)$ & $169.7(166.5-172.9)$ & 155.5 (152.2-158.8) & $-14.2(-17.5-(-10.9))$ & -1.00 \\
\hline & stayed awake & 47 & $35.0(33.9-36.2)$ & $167.6(162.6-172.7)$ & $162.4(157.3-167.5)$ & $-5.2(-9.9-(-5.2))$ & -0.30 \\
\hline Respiratory rate & stayed asleep & 149 & $33.6(33.1-34.2)$ & $56.0(53.5-58.5)$ & $47.2(44.8-49.6)$ & $-8.8(-11.7-(-5.9))$ & -0.57 \\
\hline \multirow[t]{2}{*}{ (breaths per min) } & fell asleep & 76 & $34.3(33.5-35.1)$ & $50.1(46.0-54.3)$ & $44.9(41.6-48.3)$ & $-5.2(-10.2-(-0.3))$ & -0.28 \\
\hline & woke up & 30 & $33.0(31.6-34.4)$ & $57.4(51.2-63.7)$ & $43.3(38.8-47.8)$ & $-14.1(-21.9-(-6.3))$ & -0.84 \\
\hline \multirow[t]{4}{*}{$\mathrm{SaO}_{2}(\%)$} & stayed asleep & 149 & $33.6(33.1-34.2)$ & $95.0(94.1-95.8)$ & $96.5(95.9-97.1)$ & $1.6(0.9-2.3)$ & 0.31 \\
\hline & fell asleep & 79 & $34.3(33.5-35.1)$ & $94.7(93.6-95.7)$ & $96.9(96.1-97.8)$ & $2.3(1.2-3.3)$ & 0.48 \\
\hline & woke up & 31 & $33.0(31.6-34.4)$ & $95.4(94.2-96.6)$ & $95.4(93.8-97.1)$ & $0(-1.7-1.8)$ & 0.00 \\
\hline & stayed awake & 47 & $35.0(33.9-36.2)$ & $96.3(95.1-97.6)$ & $97.3(96.4-98.3)$ & $1.0(0.1-2.0)$ & 0.23 \\
\hline
\end{tabular}




\subsubsection{Respiratory Rate}

Infants who stayed asleep showed a decrease of the respiratory rate by 8.8 breaths per $\min (95 \%$ CI 11.7-5.9) and infants who woke up a decrease by 14.1 breaths per min (21.9-6.3) during music therapy. The CIs for the baseline and post-therapy values in both groups did not overlap (Table 3 , Figure $2 b)$. The effect sizes were strongest during sleep $(-0.57)$ and in infants who woke up during therapy $(-0.84)$.

\subsubsection{Oxygen Saturation}

Oxygen saturation increased during music therapy in infants who stayed asleep (1.6\% (95\% CI 0.9-2.3\%)) and who fell asleep (2.3\% (95\% CI 1.2-3.3\%)) (Table 3, Figure 2c). Accordingly, the effect sizes were largest in these two groups ( 0.31 and 0.48 , respectively).

\section{Discussion}

This study provides the first evidence that music therapy has beneficial effects on vital signs when delivered to sleeping preterm infants. The general changes in vital signs were in line with previously published data, thus enabling stratified analyses [7,11]. We found a decrease in the respiratory rate and an increase in oxygen saturation in infants who were asleep before and after the session. Our data show that music therapy has effects on vital signs when delivered to preterm infants during sleep. While it had previously been shown that music therapy improved sleep patterns in preterm infants [11], the efficacy of music therapy had not been studied with respect to the state of wakefulness. The data from this study suggest that live music therapy can be offered to sleeping infants. This possibility largely facilitates the planning of therapy sessions.

The results of this study support previous findings on the short-term stabilization of the vital signs of preterm infants by music therapy and add new evidence that vital signs stabilize during the intervention even in sleeping infants. However, a transient stabilization of vital signs is not the main purpose of interventions in the neonatal intensive care unit. Interventions that improve the neurodevelopmental outcome at later ages and parental well-being are more important. Music therapy could have effects on the infant or parents that are not reflected by vital signs, e.g., the long-term modulation of stress levels. Parental stress and the anxiety of preterm infants receiving music therapy were lower compared to controls [15]. In line with this, former preterm infants' fear reactivity at 12 months of age and anger reactivity at 24 months of age were more similar to term-born controls after receiving music therapy in the neonatal intensive care unit compared to preterm controls who had not received music therapy [16]. Magnetic resonance imaging showed that live music therapy promoted functional brain activity and connectivity in preterm infants [17]. These studies give the first evidence that the short-term stabilization of vital signs that we and others observed might translate into a positive long-term development.

Our study has several limitations. Vital signs were not documented for the control group that received no therapy. An analysis of spontaneous changes in vital signs occurring without music therapy and during different states of wakefulness would have made exact effect size calculations possible. Music therapy sessions were performed at different times of the day and had no standardized temporal distance to feeding, causing a certain inhomogeneity of the recorded data. We did not record data on pre- and post-therapy oxygen supplementation. In our neonatal intensive care unit, oxygen supplementation is guided according to predefined target ranges of oxygen saturation depending on the corrected gestational age of an infant. Therefore, changes in oxygen saturation during the session may have been attenuated or exaggerated due to an adjustment of the oxygen supplementation. Due to the small sample size, subgroup analyses, e.g., on the interindividual response to music therapy at a given gestational age but at different corresponding postnatal ages, could not be carried out.

In conclusion, our study confirms former findings on the stabilizing effect of live music therapy on vital signs. It adds new evidence that music therapy is effective during sleep 
in preterm infants, making the planning of therapy sessions easier for music therapists, nurses, and parents.

Author Contributions: Study design: N.B., M.V.D., U.F.-M., S.K.; delivery of music therapy: S.K. and M.D.; statistical analyses: N.B. and A.-K.D., figures: N.B.; drafting of manuscript: S.K., critical revision: N.B., B.H., M.V.D., U.F.-M., A.-K.D. All authors have read and agreed to the published version of the manuscript.

Funding: The study received funding from the Stiftung Universitaetsmedizin Essen. M.V.D. and N.B. received funding from the Medical Faculty of the University of Duisburg-Essen (IFORES).

Institutional Review Board Statement: The study was approved by the local ethics committee of the Medical Faculty of the University of Duisburg-Essen (18-8035-BO). Registration Number for clinical research: DRKS00025753.

Informed Consent Statement: Written informed consent was obtained from the participants' parents or legal guardians before inclusion into the study.

Data Availability Statement: Original data will be made available to any qualified researcher upon request.

Conflicts of Interest: The authors have no conflict of interests to declare.

\section{References}

1. Parikh, N.A. Advanced neuroimaging and its role in predicting neurodevelopmental outcomes in very preterm infants. Semin. Perinatol. 2016, 40, 530-541. [CrossRef] [PubMed]

2. Moon, C.M.; Fifer, W.P. Evidence of Transnatal Auditory Learning. J. Perinatol. 2000, 20, S37-S44. [CrossRef] [PubMed]

3. Haslbeck, F.; Noecker-Ribaupierre, M.; Zimmer, M.-L.; Schrage-Leitner, L.; Lodde, V. Musik von Anfang an. Referenzrahmen zur Anwendung von Musiktherapie in der Neonatologie. Deutsche gesellschaft für Musiktherapie. 2017. Available online: https:// www.musiktherapie.de/wp-content/uploads/2019/05/Referenzrahmen-Fachkreis-Musiktherapie-Neonatologie.pdf (accessed on 21 June 2021).

4. Ghetti, C.M.; Vederhus, B.J.; gaden, T.S.; Brenner, A.K.; Bieleninik, Ł.; Kvestad, I.; Assmus, J.; gold, C. Longitudinal Study of Music Therapy's Effectiveness for Premature Infants and Their Caregivers (LongSTEP): Feasibility Study with a Norwegian Cohort. J. Music. Ther. 2021, 58, 201-240. [CrossRef] [PubMed]

5. Van Dokkum, N.H.; Jaschke, A.C.; Ravensbergen, A.-G.; Reijneveld, S.A.; Hakvoort, L.; De Kroon, M.L.A.; Bos, A.F. Feasibility of Live-Performed Music Therapy for Extremely and Very Preterm Infants in a Tertiary NICU. Front. Pediatr. 2020, 8, 581372. [CrossRef] [PubMed]

6. Anderson, D.E.; Patel, A.D. Infants born preterm, stress, and neurodevelopment in the neonatal intensive care unit: Might music have an impact? Dev. Med. Child Neurol. 2018, 60, 256-266. [CrossRef]

7. Bieleninik, Ł.; ghetti, C.; gold, C. Music Therapy for Preterm Infants and Their Parents: A Meta-analysis. Pediatrics 2016, 138, e20160971. [CrossRef]

8. Hartling, L.; Shaik, M.S.; Tjosvold, L.; Leicht, R.; Liang, Y.; Kumar, M. Music for medical indications in the neonatal period: A systematic review of randomised controlled trials. Arch. Dis. Child. Fetal Neonatal Ed. 2009, 94, F349-F354. [CrossRef]

9. Haslbeck, F.B. Music therapy for premature infants and their parents: An integrative review. Nord. J. Music. Ther. 2012, 21, 203-226. [CrossRef]

10. Standley, J. Music Therapy Research in the NICU: An Updated Meta-Analysis. Neonatal Netw. 2012, 31, 311-316. [CrossRef] [PubMed]

11. Loewy, J.; Stewart, K.; Dassler, A.-M.; Telsey, A.; Homel, P. The Effects of Music Therapy on Vital Signs, Feeding, and Sleep in Premature Infants. Pediatrics 2013, 131, 902-918. [CrossRef] [PubMed]

12. Arnon, S.; Shapsa, A.; Forman, L.; Regev, R.; Bauer, S.; Litmanovitz, I.; Dolfin, T. Live Music Is Beneficial to Preterm Infants in the Neonatal Intensive Care Unit Environment. Birth 2006, 33, 131-136. [CrossRef]

13. Mirmiran, M.; Maas, Y.G.H.; Ariagno, R.L. Development of fetal and neonatal sleep and circadian rhythms. Sleep Med. Rev. 2003, 7, 321-334. [CrossRef] [PubMed]

14. Maier-Riehle, B.; Zwingmann, C. Effektstaerkevarianten beim Eingruppen-Prae-Post-Design: Eine kritische Betrachtung. Rehabilitation 2000, 39, 189-199. [CrossRef] [PubMed]

15. Kehl, S.M.; La Marca-Ghaemmaghami, P.; Haller, M.; Pichler-Stachl, E.; Bucher, H.U.; Bassler, D.; Haslbeck, F.B. Creative Music Therapy with Premature Infants and Their Parents: A Mixed-Method Pilot Study on Parents' Anxiety, Stress and Depressive Symptoms and Parent-Infant Attachment. Int. J. Environ. Res. Public Health 2020, 18, 265. [CrossRef] 
16. Lejeune, F.; Lordier, L.; Pittet, M.P.; Schoenhals, L.; grandjean, D.; Hueppi, P.S.; Filippa, M.; Tolsa, C.B. Effects of an Early Postnatal Music Intervention on Cognitive and Emotional Development in Preterm Children at 12 and 24 Months: Preliminary Findings. Front. Psychol. 2019, 10, 494. [CrossRef] [PubMed]

17. Haslbeck, F.B.; Jakab, A.; Held, U.; Bassler, D.; Bucher, H.-U.; Hagmann, C. Creative music therapy to promote brain function and brain structure in preterm infants: A randomized controlled pilot study. NeuroImage Clin. 2020, 25, 102171. [CrossRef] [PubMed] 